Published in final edited form as:

Trends Cancer. 2016 October ; 2(10): 606-618. doi:10.1016/j.trecan.2016.09.001.

\title{
The Emerging Role of Cdk5 in Cancer
}

\author{
Karine Pozo ${ }^{1,2}$ and James A. Bibb ${ }^{3}$ \\ ${ }^{1}$ Department of Psychiatry, The University of Texas Southwestern Medical Center, Dallas, TX \\ USA \\ ${ }^{2}$ Department of Surgery, The University of Texas Southwestern Medical Center, Dallas, TX USA \\ ${ }^{3}$ Department of Surgery, The University of Alabama Birmingham, Birmingham, AL USA
}

\begin{abstract}
Cdk5 is an atypical cyclin-dependent kinase that is well characterized for its role in the central nervous system rather than in the cell cycle. However Cdk5 has been recently implicated in the development and progression of a variety of cancers including breast, lung, colon, pancreatic, melanoma, thyroid and brain tumors. This broad pro-tumorigenic role makes $\mathrm{Cdk} 5$ a promising drug target for the development of new cancer therapies. Here we review the contribution of Cdk5 to molecular mechanisms that confer upon tumors the ability to grow, proliferate and disseminate to secondary organs, as well as resistance to chemotherapies. We subsequently discuss existing and new strategies for targeting Cdk5 and its downstream mechanisms as anti-cancer treatments.
\end{abstract}

\section{Keywords}

Cdk5; cancer; resistance

\section{Trends Box}

-

Cdk5 is not mutated in cancer tissues but its expression and activity are deregulated. Because discovery of the molecular causes of cancer is predominantly based on the identification of tumor-associated mutations, the role of in cancer has been overlooked until recently.

Cdk5 contributes to tumor proliferation, migration, angiogenesis, and is linked to chemotherapy resistance and anti-tumor immunity

Strategies to target Cdk5 and downstream pathways include small molecules to block the kinase activity and peptides to disrupt Cdk5 binding to its activators or substrates.

Corresponding authors: Bibb, J.A. (jbibb@uab.edu), Pozo, K (Karine.Pozo@utsouthwestern.edu).

Publisher's Disclaimer: This is a PDF file of an unedited manuscript that has been accepted for publication. As a service to our customers we are providing this early version of the manuscript. The manuscript will undergo copyediting, typesetting, and review of the resulting proof before it is published in its final citable form. Please note that during the production process errors may be discovered which could affect the content, and all legal disclaimers that apply to the journal pertain. 
Genetically modified animal models of cancer may be generated by deregulating Cdk5 via overexpression of its activator, p25, in cancer cells. Such models may be clinically relevant and exhibit tumors that grow within a physiological microenvironment in an animal with an intact immune system.

\section{Cdk5: an overlooked player in the cancer field}

Deregulation of the cell cycle is a fundamental process that underlies cancer proliferation [1]. Progression through the cell cycle is regulated by the coordinated actions of cyclindependent kinases (Cdks), which are, therefore, targets-of-interest for anti-cancer drugs. However many neoplasia are currently not responsive to anti-Cdk therapies [2]. The protein kinase, Cdk5 has long been overlooked in cancer-related studies because it is an atypical $\mathrm{Cdk}$ that is predominantly expressed in neurons and is mainly activated by the non-cyclin activators, p35, or its truncated product, p25 [3] (Box 1) (Figure 1b, Key Figure). Nevertheless, $\mathrm{Cdk} 5$ is rapidly emerging as a new player in tumorigenesis with functions ranging from cell proliferation to metastatic invasion (Figure 1c, Key Figure) and angiogenesis. Here we review recent evidence demonstrating that Cdk5 plays a critical role in the molecular mechanisms driving tumor formation and development. We further examine new studies indicating a link between Cdk5 and radio-/chemotherapy resistance as well as Cdk5 and immunotherapy. Finally we discuss why Cdk5 may be a valid target for anticancer therapies.

\section{Box 1}

\section{Cdk5, an unconventional cyclin-dependent kinase}

Although Cdk5 exhibits approximately $60 \%$ amino acid sequence identity with Cdk 1 and Cdk2 [3], it is highly expressed in brain compared to other organs, and is therefore considered as a neuronal kinase. $\mathrm{Cdk} 5$ has been well-characterized for brain development, function and pathologies [3], while roles in non-neuronal tissues have been under-explored until recently [22,63].

Control of Cdk5 activity is unlike that of any other Cdks [3]. It is activated by binding to the non-cyclin activators, $\mathrm{p} 35$ or p39, and phosphorylates substrates on serine or threonine residues within the consensus motif S/TPXH/K/R (Figure 1b, 1d, Key Figure). Cdk5 activation by cyclins D, E, G1 and the non-cyclin protein, p67 has also been reported but the significance in tumorigenesis has not been elucidated yet [64]. Cdk5 can also be activated by binding to cyclin I [65] and in cervical cancer patients the Cdk5/ cyclin I complex is associated with resistance to cisplatin treatment [66]. Since the predominant Cdk5 activators are p35 and p39, these are discussed further here. Cdk5/p35 and Cdk5/p39 are constitutively active forms of the kinase, with relatively low catalytic efficiency compared to more acutely activated kinases, and are distributed throughout cells. Cleavage of Cdk5 cofactors by calpain removes the first 100 amino acids, to produce $\mathrm{p} 25$ or $\mathrm{p} 29$, which still contain the Cdk5 binding domain. The resulting Cdk5/p25 or Cdk5/p29 holoenzymes have longer half-lives and altered substrate 
specificity compared to $\mathrm{Cdk} 5 / \mathrm{p} 35$ or $\mathrm{Cdk} 5 / \mathrm{p} 39$. Cdk5/p35 is often docked to cell membranes due to a myristoylation in the p35 N-terminal region. Loss of this domain as a result of cleavage renders Cdk5/p25 more soluble, able to access cytoplasmic and nuclear substrates that are not in cellular membranes proximity [67] (Figure 1, Key Figure). Cdk5/p25 and Cdk5/p35 have been linked to tumorigenesis, however Cdk5/p25 seems to be a more toxic form linked to neurodegenerative diseases and paradoxally, pro cell cycle [22]. Regulation of calpain-mediated p35-to-p25 cleavage in cancer cells has not been studied. It can be speculated that environmental stresses cause changes in calcium concentration that activates the calpain protease activity. P35 also undergoes post-translational modifications in addition to proteolytic cleavage, including autophosphorylation, which may impact regulatory mechanisms [67]. The cytoskeleton protein, nestin, regulates the turnover of p35 into p25 in myoblasts [68]. A similar mechanism might occur in cancer cells. Getting a better insight of the mechanisms underlying the p35-to-p25 cleavage may offer new targets for Cdk5-driven cancer treatments.

\section{Human cancers express Cdk5}

Expanding evidence at the gene, mRNA and protein levels supports a role for Cdk5 in human cancers (Table 1) and its expression and activity as biomarkers for the prediction of cancer severity. First, genetic variations such as the amplification of the Cdk5 gene [4] or p35/p39 genes [5, 6] as well as single-nucleotide polymorphism (SNP) in the Cdk5 gene promoter region [7] occur in several cancer populations. These are, for example, associated with higher lung cancer risks in the Korean population $[4,7]$ and with aggressive forms of prostate cancers in African-Americans [8]. Second, mRNA and protein expression levels of Cdk5 and its activators are increased, or decreased, in several forms of cancer and these alterations are correlated with cancer severity (Figure 1a, Key Figure; Table 1). For example, Cdk5 and p35/p25 are elevated in pulmonary neuroendocrine cancers [9-11], in sporadic and familial forms of medullary thyroid carcinoma (MTC) [12] and pituitary adenoma [13]. In fact Cdk5 and p35/p25 expression appear to typify neuroendocrine cancer pathology. In tissues from non-small cell lung cancer (NSCLC), breast, brain and nasopharyngeal cancer patients, increased Cdk5 and/or activator expression parallels advanced cancer stages, occurrence of lymph node metastasis, and overall poor 5-year survival, while low Cdk5 levels correlates with metastatic-free disease [14-19]. Somewhat remarkably abnormal low Cdk5 or activator tumor levels are also indicative of poor prognosis as in advanced gastric cancer [20] or hepatocellular carcinoma (HCC) [21]. More studies are needed to understand the molecular mechanisms by which $\mathrm{Cdk} 5$ and activators contribute to these cancers. Below, we discuss what is currently known about Cdk5 role in oncogenic pathways.

\section{Molecular links between Cdk5 and the cell cycle}

Historically, Cdk5 has not been considered as a potential cell cycle regulator because it is predominantly expressed in non-dividing, post-mitotic neurons [3]. However it has been noted that Cdk5 facilitates amyloid $\beta$ protein-induced cell cycle re-entry in neurons of mouse models of Alzheimer's disease [22] and directly modulates the function of tumor 
suppressors and transcription factors (Figure 2a; Table 2). This raises the possibility that evolution of CNS function occurred through adaption of cell cycle mechanisms. Thus Cdk5 may contribute to neurodegeneration and oncogenesis through overlapping or common pathways and especially those related to the cell cycle and cell proliferation (Figure 1c, Key Figure).

\section{Cdk5 and the Rb/E2F pathway}

Cdk5 modulates the retinoblastoma protein $(\mathrm{Rb}) / \mathrm{E} 2 \mathrm{~F}$ pathway by phosphorylating $\mathrm{Rb}$ [23], thereby promoting cancer cell proliferation as in MTC [12]. Rb controls the transition from the G0/G1 to S-phase and thus the initiation of the cell cycle by sequestering the transcription factor E2F [22] (Figure 2a). It is well accepted that upon Rb phosphorylation by Cdk4-6/cyclin D, E2F is released and activates the transcription of genes required for cell cycle progression. In MTC, phosphorylation of Rb by Cdk5 results in E2F target genes expression, including Cdk2, $\mathrm{p} 15^{\mathrm{INK} 4 \mathrm{~b}}$ and $\mathrm{p} 21^{\mathrm{CIP} / \mathrm{WAF} 1}$ with cancer proliferation ensuing [12]. All these events are prevented by Cdk5 inhibition, supporting a key role for Cdk5 activity in MTC via Rb dysregulation. These provocative findings also raise the intriguing possibility that $\mathrm{Cdk} 5$ is capable of replacing Cdk4/6 in certain cancer types, providing a molecular basis for why some cancers are resistant to Cdk4/6 inhibitors. Whether Cdk5 regulates the function of Rb-like proteins, p107 and p130, which are also E2F-binding tumor suppressors, in Rb-negative tumors remains to be determined. Moreover, it would be interesting to find out if $\mathrm{Cdk} 5$ can modulate cell cycle progression by associating with E2F in a p35-dependent manner thereby interfering its function as observed in neurons [22]. Finally, understanding the cell cycle progression effects of Cdk5 phosphorylation of E2F targets genes, p19 ${ }^{\text {INK4d }}$ and the Cdc25 family of dual-specific phosphatases, Cdc25A-C on $[24,25]$ would yield more insights into the role of Cdk5 in the cell cycle and tumorigenesis.

\section{Cdk5 and STAT3}

Besides Rb/E2F, Cdk5 has been associated with the dysregulation of signal transducer and activator of transcription 3 (STAT3) in cancer cells. This transcription factor integrates a variety of signaling pathways and controls the expression of cell cycle genes such as cyclin D1 and c-Fos [26] (Figure 2a). In normal cells, STAT3 becomes activated following cell stimulation by a cytokine or growth factor, while in cancer cells STAT3 is often deregulated. Cdk5 interacts with and phosphorylates STAT3 at Ser-727, which is necessary for MTC and prostate cancer cells proliferation [27, 28]. STAT3-target genes are indeed induced upon Cdk5 activation. Importantly, prostate cancer cells and prostate cancer patient tumors express Cdk5, p35 and phosphorylated Ser-727 STAT3 [28, 29]. It would be interesting to define the determinants for Cdk5-dependent phosphorylation of Rb versus STAT3.

\section{Cdk5 and the Androgen Receptor}

In prostate cancer, $\mathrm{Cdk} 5$ can also phosphorylate the transcription factor, androgen receptor (AR) (Figure 2a). Cdk5 interacts with AR and phosphorylates it at Ser-308, thereby stabilizing the receptor and enabling transcription of AR-induced genes in early stage prostate cancer cells [30]. AR phosphorylation at Ser-81 by Cdk5 also promotes the growth of prostate cancer cells and prostate tumor xenografts [28]. AR may also be stabilized by interacting with a Cdk5-phosphorylated form of STAT3, i.e. Ser-727 STAT3 in prostate 
cancer cells, thus providing a mechanism linking Cdk5-STAT3-AR in prostate cancer tumorigenesis [28]. Interestingly, AR gene expression in hormone therapy-resistant prostate cancers is driven by the $\mathrm{Rb} / \mathrm{E} 2 \mathrm{~F}$ pathway upon $\mathrm{Rb}$ genetic inactivation. It would be interesting to determine if Cdk5 plays a role in the signaling mechanisms underlying advanced forms of prostate cancers by regulating AR or E2F function.

Overall, Cdk5 appears to modulate the function of cell cycle proteins, including tumor suppressors and transcription factors, thereby regulating cancer cell proliferation.

Interestingly Cdk5 has previously been proposed as a cell cycle suppressor in neurons [22]. Indeed, in gastric cancer cells, Cdk5 accumulation in the nucleus has an anti-tumor effect, while decrease in nuclear Cdk5 correlates with cancer progression [20]. We speculate that for some malignancies, Cdk5 may have a protective effect and act as a cell suppressor as found in neurons, while in others, dysregulated Cdk5 promotes tumorigenesis.

\section{Cdk5 in DNA repair and drug resistance}

Increasing evidence indicates that $\mathrm{Cdk} 5$ contributes to the initiation of the DNA damage response (DDR) and DNA repair. Upon exposure to environmental stressors such as UV, genotoxic agents or radiation, these mechanisms are activated and ensure maintenance of genome integrity during cell division by preventing the transmission of damaged DNA. Deregulation of the DDR and DNA repair have been associated with tumorigenesis and resistance to conventional DNA-damaging agent-based cancer treatments, such as chemoand radiation-therapies [31]. As discussed below, Cdk5 role in the DDR and DNA repair is leading to the exciting hypothesis that Cdk5 inhibition may be a valid strategy to bypass chemo- and radiation-therapy resistance.

\section{DNA damaging agents induce Cdk5}

Cdk5 is activated in cancer cells or tissues exposed to conventional DNA-damaging therapies including ionizing radiations (IR) or genotoxic agents, such as topoisomerase inhibitors and DNA cross-linkers [16, 32-35] (Figure 2b). Upregulation of p35 expression, via the Egr1 promoter, and formation of p25 may underlie increases in Cdk5 activity in IRexposed glioblastoma (GBM) and genotoxic agent-treated neuroblastoma [17, 36, 37]. IR/ genotoxic agent-induce Cdk5 activation likely contributes to DNA repair as DNA double strand breaks (DSBs) are more abundant following pharmacological inhibition of Cdk5 or si/ shRNA-mediated knock-out of Cdk5 in cancer cells exposed to DNA-damaging agents [3335].

\section{Cdk5 and the regulation of the DDR and DNA repair}

Cdk5 is known to activate cell cycle checkpoints, which is a pre-requisite to DNA repair (Figure 1c, Figure 2b). First, Cdk5 phosphorylates the checkpoint-activating kinase Ataxiatelangiectasia mutated (ATM) at Ser-794 to switch on its kinase activity [34, 35, 37] in some cancer cells. Consistent with these findings, Cdk5 activity blockade in HCC cell lines prevents ATM phosphorylation and the initiation of the downstream DDR signaling cascade, while the formation of DSBs is increased [34]. Second, Cdk5 phosphorylates and activates replication protein A (RPA)-32, which is a necessary step for the induction of the intra-S 
phase checkpoint and DNA repair [16]. Finally, Cdk5 transduces the signals necessary for the expression of DNA repair molecules. For example, expression of the DNA repair endonuclease, Eme1, in genotoxic agent-treated colorectal cancer cell lines is dependent on the activation of STAT-3 by Cdk5 [33, 38]. It will be useful to determine if Cdk5's role in the initiation and transduction of the DDR is dependent on the cancer type, the type of DNA-damaging method, or on the severity of the DNA damage, as this might influence potential treatment strategies.

\section{Cdk5 and clinical implications of resistance to DNA damaging therapies}

Cdk5 function in the DDR and DNA repair may be linked to resistance to DNA-damaging anti-cancer therapies. Cdk5 has indeed been implicated in resistance to PARP inhibitors [16, $32,34,39]$, topoisomerase inhibitors and DNA crosslinkers. In line with these observations, treatment of the NSCLC cell line, A549, the proliferation of which is driven by Cdk5 [11], with the Cdk5 inhibitor, roscovitine, enhanced its sensitivity to IR [40]. In all cases, Cdk5 inhibition or Cdk5 knock-down restored chemotherapeutics sensitivity. Likewise, the antitumor response to the chemotherapeutic in a xenograft mouse model of HCC was improved when roscovitine was co-administered [34]. These observations all suggest that effective Cdk5 inhibitors could be administered in combination with DNA-damaging agents to improve conventional anti-cancer treatments.

\section{Cdk5 and cancer dissemination}

During tumorigenesis, malignant cells can acquire phenotypic features enabling them to leave the primary tumor and transit via the blood and lymphatic circulation to secondary sites, in which they will grow into macrometastases [1]. In addition to tumor proliferation, Cdk5 is involved in the signaling pathways that underlie cancer cell migration to metastatic sites. Cdk5 has been linked to different components of the cancer dissemination process (Figure 2c).

\section{Cdk5 and "migration-proliferation dichotomy"}

Cdk5 contributes to the early events that decide whether tumor cells will continue growing or migrate towards secondary tumor sites for metastatic invasion [41]. When breast cancer cells are stimulated with EGF, Cdk5 becomes activated and phosphorylates the $\mathrm{Ga}$ interacting vesicle associated protein, Girdin, which in turn activates traditional downstream G-coupled receptor-dependent signaling mechanisms that promote cell migration. Thus Cdk5 activity may be key in determining whether cells maintain a strictly proliferative versus invasive agenda.

\section{Cdk5 and epithelial-to-mesenchymal transition}

Acquisition of the invasive phenotype requires cancer cells to change their cell-cell adhesion properties and acquire a motility phenotype, a process called epithelial-to-mesenchymal transition EMT (Figure 3). In breast cancer, Cdk5 is essential for initiation of metastatic invasion upon stimulation with transforming growth factor $\beta 1$ (TGF $\beta 1$ ) [15]. Depleting Cdk5 is sufficient to abolish TGF $\beta 1$-induced EMT. Interestingly, expression of Cdk5 and 
p35 in breast cancer cells is enhanced upon TGF $\beta 1$ stimulation. It will be useful to determine how TGF $\beta 1$ regulates the transcription of Cdk5 and $\mathrm{p} 35$.

\section{Cdk5 and cancer cell migration}

Cdk5 activity drives the migration and metastatic invasion of some forms of lung [42], prostate [29, 43], pancreas [6, 44], melanoma [45], pituitary [13] and thyroid [27] cancers. The migration of these cancer cells in culture or in vivo is stopped upon Cdk5 inhibition (Figure 2c). Mechanistically, Cdk5 activity is dependent on p35 expression, which is controlled by the transcription factor, human achaete-scute homolog 1 (hASH1) in migrating neuroendocrine lung cancer cells [42].

In pancreatic cancer, $\mathrm{Cdk} 5$ is downstream of $\mathrm{K}$-Ras [6, 44]. In fact, K-Ras promotes the generation of p25 and activation of Cdk5, which subsequently turns on the Ral pathway and causes changes in cell morphology favoring cell migration. PDAC cells exhibit shorter processes and become flat upon Cdk5 inhibition downstream of Ras [6]. In fact Cdk5 activity is associated with the invasive cellular phenotype, including the remodeling of the actin-cytoskeleton, the formation of invadopodia and the regulation of focal adhesions, which are protein complexes involved in cell-extracellular matrix adhesions and motility (Box 2). Whether Cdk5 involvement in cancer cell migration is dependent on cancer context or is common to all cancer types remains to be elucidated.

\section{Box 2}

\section{Cdk5 role in cytoskeleton regulation and paclitaxel resistance}

Cdk5 is implicated in the acquisition of the invasive phenotype via regulation of actin and microtubule cytoskeleton as well as focal adhesions, which underlie the formation of invadopodia for cell motility (Table 2). Cdk5 regulates the formation of F-actin bundles in breast cancer cells [15]. Furthermore, Cdk5 modifies actin dynamics regulators such as caldesmon, an actin- and calmodulin-binding protein that participates in the migration and metastatic invasion of melanoma and breast cancer cells [45]. Cdk5 has long been implicated in the regulation of microtubule complexes that are essential for cell motility. For example, the microtubule-associated proteins, Tau, $[69,70]$ and Collapsin Response Mediator Protein-2 (CRMP-2) [71], which promote microtubule assembly, as well as the microtubule-disassembly protein, Stathmin [72,73], are well-characterized Cdk5 substrates implicated in cancer progression. Finally Cdk5 contributes to the formation and stabilization of focal adhesions, which serve as anchor for the migration process, by phosphorylating talin and focal adhesion kinase (FAK) in migrating cells $[6,15,74]$. Cdk5's role in cytoskeleton remodeling may underlie resistance mechanisms to paclitaxel, a common chemotherapy agent that acts by preventing microtubule depolymerization and thus mitosis. Paclitaxel resistance is common and is associated with an invasive phenotype, which can be prevented by blocking Cdk5 activity [75]. Consistently the sensitivity of ovary cancer cells to paclitaxel is enhanced upon Cdk5 depletion [76]. Remarkably this drug induces Cdk5 expression, thereby promoting cell migration [61]. Thus, combinatorial treatments with a Cdk5 inhibitor and paclitaxel may prevent paclitaxel resistance [61]. Interestingly, Tau, was identified as a potential 
predictive biomarker of the response of breast and gastric cancer patients to paclitaxel $[70,77]$ with Tau-negative patients likely to have a better response to the drug.

\section{An unexpected role for Cdk5 in tumor angiogenesis}

One of the least suspected functions for Cdk5 is its critical contribution to angiogenesis. This process by which blood vessels form from pre-existing ones to ensure the delivery of oxygen and nutrients to growing tumors, allows removal of metabolic wastes and, together with lymphatic vessels, provide an escape route for cancer cells migration to metastatic sites. Evidence is rapidly implicating Cdk5 as a master regulator of angiogenesis at least in some cancers [46]. In addition to tumor cells, Cdk5 is expressed in endothelial cells lining of blood vessels that are usually quiescent [47] and regulates their proliferative and migratory properties [48, 49] (Figure 2c).

\section{Cdk5 and endothelial cell survival}

Cdk5 is critical for endothelial cell proliferation and disruption of Cdk5 expression causes apoptosis. Cdk5 protein levels are elevated in proliferating cultured bovine aortic endothelial cells while Cdk5 expression is lower in quiescent cells [47]. Consistently overexpression of Cdk5 (and Cdk2) triggers cell proliferation and angiogenesis whereas Cdk5/2 inhibition with roscovitine arrests both processes and leads to apoptosis [47, 50].

\section{Cdk5 and endothelial cell migration}

Cdk5 also contributes to endothelial cell migration [48, 49]. Cdk5 regulates lamellipodia formation and thus endothelial cell migration by remodeling the actin cytoskeleton via regulation of the monomeric GTPase, Rac1 $[48,51]$. In melanoma xenograft mouse models, Cdk5 inhibition prevents formation of functional blood vessels and consequently tumor growth [49].

\section{Cdk5 and angiogenic molecules}

Several lines of evidence suggest there is an intricate relationship between Cdk5 and angiogenic molecules. First, Cdk5 expression in endothelial cell is induced by proproliferative, angiogenic factors, including basic fibroblast growth factor (bFGF), that are secreted by tumor cells [50], while angiogenesis inhibitors such as angiostatin prevent Cdk5 expression and cause apoptosis [47]. Second, Cdk5 regulates the expression and activity of the transcription factor, hypoxia-inducible factor 1a (HIF1a) both in HCC tumor and endothelial cells (Figure 2c, d). Thus Cdk5 exerts direct control over expression of the HIF1a target gene, vascular endothelial growth factor (VEGF)-A and VEGF receptor 1, which are essential for the formation of new blood vessels in tumors [46]. Interestingly, $\mathrm{Cdk} 5$ can also act at the tumor cell level, inducing secretion of pro-angiogenic molecules. It will be critical in the future to determine if a regulatory loop between tumor and endothelial cells exists to control angiogenesis and how Cdk5 fits into it. 


\section{Cdk5 as an anti-angiogenic drug target}

The apparent role of Cdk5 in endothelial cell physiology and tumor angiogenesis suggests it as a target for anti-angiogenic treatment. New anti-Cdk5 drugs are being developed for this purpose [51]. Furthermore other potential downstream targets of Cdk5 with funtion in angiogenesis, such as HIF1a and Notch, are being identified [46, 49]. With the recent discovery that Cdk5 is involved in the development of lymphatic vessels [52], it is becoming clear that Cdk5 plays a more important role than suspected in the tumor vasculature and in supporting tumor development and spread.

\section{Current strategies for Cdk5 targeting}

From pan-Cdk inhibitors to pathway-specific blocking peptides, the repertoire of Cdk5targeting drugs is increasing [2, 53] (Figure 1d-1f, Key Figure). While currently available compounds and agents have limitations, there is now renewed interest in the therapeutic targeting of $\mathrm{Cdk} 5$ or its pathogenic effectors.

\section{Small molecules inhibitors}

Inhibition of kinase activity using pan-Cdk small molecules inhibitors is the most common strategy currently on hand to target Cdk5 in preclinical studies. For some of these drugs, clinical trials have been conducted with mixed results [2]. Roscovitine is widely used to inhibit Cdk5 in cell lines and mouse models. This purine analog competes for ATP-binding to Cdk5, but also inhibits Cdk1, 2, 9, 7 [54]. Roscovitine has no effects on Cdk4/6, rendering it useful to study $\mathrm{Cdk} 5$ effects on $\mathrm{Rb}$ and the cell cycle. Although roscovitine exhibits anticancer properties preclinically, clinical trials have been inconclusive to date [2]. In contrast dinaciclib, which has improved potency for Cdk5 and Cdk1, 2, 9, exhibits anti-proliferative effects on hematological cancers during clinical trials [2,53]. Thus targeting Cdk5 along with Cdk1, 2, 9 may be a valid anti-cancer strategy. Other small molecule inhibitors CP681301 [55], indolinone A [56] and PJB [45] exhibit increased selectivity for Cdk5 and Cdk2 over other Cdks and anti-cancer activity in cell lines [12, 45], but in vivo preclinical studies remain to be conducted. Thus more inhibitors are available to target Cdk5 more specifically.

\section{A peptide for the disruption of Cdk5/activator binding}

A peptide derived from $\mathrm{p} 35 / \mathrm{p} 25$ that selectively blocks Cdk5/p25 interactions has been used to rescue the Cdk5/p25-induced pathological phenotypes [53] (Figure 1e, Key Figure). However the anti-cancer effect of this peptide remains to be determined. A peptide that disrupts Cdk5/p35 binding would be a useful tool to target Cdk5 in tumor cells or animal models because both holoenzymes, $\mathrm{Cdk} 5 / \mathrm{p} 35$ and $\mathrm{Cdk} 5 / \mathrm{p} 25$, appear to be tumorigenic.

\section{Small interfering peptides: targeting Cdk5/substrate interactions}

An alternative approach for preventing Cdk5-induced pathogenesis consists in using short peptides to selectively block Cdk5-dependent phosphorylation of aberrant substrate within a given pathogenic pathway (Figure 1e, Key Figure). These $\sim 20$ amino-acid peptides, designed based on the Cdk5 phosphorylation site on the substrate, were successful in selectively preventing Cdk5 phosphorylation of Rb, NR2B and PDE4 to impair molecular 
processes leading respectively to cancer progression, loss of cognition or depression [12, 57, 58]. Using pathway-specific peptides has the advantage of limiting side-effects due to mechanistic specificity, although size and stability issues are of concern. Rigorous preclinical studies will be necessary to confirm the possibility of using these peptides or peptide-like molecules as anti-cancer treatments.

\section{Drug combinations}

Combining Cdk5 inhibitors with other drugs is appearing as an attractive strategy for improving existing therapies. Co-treatment of roscovitine and irrinotecan arrests HCC growth in mouse models [34]. Combining Cdk5 inhibition with the antiviral agent, tilorone, selectively inhibited growth, proliferation and invasive phenotype of prostate cancer cells [59], while using the Cdk5 inhibitor AC1MMYR2 in combination with the chemotherapy agent, Paclitaxel may prevent resistance associated with the use of Paclitaxel [60, 61].

\section{Concluding Remarks}

Cdk5 appears to play a central role in tumorigenic pathways and therefore may be a valid target for anti-cancer drugs. Yet, many challenges remain (see Outstanding Questions Box). In particular current clinical trials with pan-Cdk inhibitors have not been entirely conclusive. Developing more potent Cdk5 inhibitors and targeting Cdk5 downstream pathways in addition to Cdk5 may be a more effective strategy to stop cancer progression and limit adverse events.

\section{Outstanding Questions Box}

- $\quad \mathrm{Cdk} 5$ can exist as both $\mathrm{p} 35 / \mathrm{Cdk} 5$ and $\mathrm{p} 25 / \mathrm{Cdk} 5$ complexes in cancer tissues. These two holoenzymes have different enzymatic properties with $\mathrm{p} 25 / \mathrm{Cdk} 5$ being apparently more pathogenic. How is $\mathrm{p} 25$ generated in cancer cells? What cues activate calpain cleavage of p35 into p25 in cancer cells? What is the contribution of nestin in the p35to-p25 turnover? Can the $\mathrm{p} 35 / \mathrm{Cdk} 5$ over $\mathrm{p} 25 / \mathrm{Cdk} 5$ ratio determine cancer prognosis? What are the exact roles of Cdk5/p35 and Cdk5/p25 in angiogenesis? How do they affect the secretion of pro-angiogenic growth factors by tumors and in which conditions?

- $\quad \mathrm{Cdk} 5$ is involved in many tumorigenic processes in different type of cancers. Are all cancers somehow dependent on Cdk5 activity at one stage of their development?

- $\quad$ Given that Cdk5 is linked to resistance to DNA-damaging cancer drugs and paclitaxel, can Cdk5, p35, p25 or downstream signaling effectors be used as biomarkers to predict patient response to conventional cancer therapies and to stratify cancer patient population for personalized medicine treatments?

- New potent and specific targeted therapies are being developed to inhibit Cdk5 or its downstream effectors and display anti-tumor effects in cell culture and preclinical models. Will these compounds have anti- 
cancer effects in clinical trials when used as mono- or combinatorial therapies? Will combinatorial treatments of Cdk5 inhibitor and DNAdamaging agents/paclitaxel sensitize drug resistant tumors in human as observed preclinically? What will be the adverse events?

Cdk5 regulates the expression of the immune checkpoint PD-L1 in medulloblastoma. Will targeting Cdk5 be a valid immunotherapy strategy in other cancers?

- Drugs targeting epigenetic mechanisms are showing promising results in cancer treatments. Would targeting Cdk5 epigenetic regulation be a better anti-cancer strategy than targeting Cdk5 kinase activity?

Generating animal models of different cancers will be crucial to pinpoint the neoplastic roles of Cdk5 and identify targets for the treatment of Cdk5-driven cancers as well as for preclinical testing new therapies. Conditional expression of p25 under the appropriate promoter appears as a valid strategy to develop clinically relevant Cdk5-driven cancers that recapitulate the human disease. In this new era of immunotherapy, using animals in which tumor grows in a physiological microenvironment and in the context of a functional immune system, may be crucial to obtain results that can be reproducible in patients.

Identifying the population of patients who could benefit from Cdk5-based treatments may also improve the outcomes of clinical trials. Defining a panel of biomarkers that includes Cdk5, activators and phosphorylation sites on pro-neoplastic effectors would allow stratification of the patient population, facilitating more precise treatments.

Knowledge gained in the field of neuroscience over the past 2-3 decades has given cancer researchers hints on the oncogenic functions of Cdk5 and have led to potential translational applications that may benefit cancer patients. For example, the links between Cdk5 and resistance to conventional therapies, i.e. DNA-damaging and microtubule-stabilizing agents, open new treatment opportunities. An important next challenge will be to determine in the clinic if combining Cdk5 inhibitors can improve tumor-resistant patient responses to conventional treatments, as observed in cell culture and preclinical models. Furthermore, the groundbreaking finding that $\mathrm{Cdk} 5$ regulates the expression of the immune checkpoint programmed cell death-ligand 1 (PD-L1) [62] may offer new applications for Cdk5-targeting drugs in the immunotherapy.

Recent efforts have focused on targeting Cdk4/6 [2] and have led to the development and FDA-approval of potent inhibitors for the treatment of some breast cancers. However resistance to these drugs are frequently observed. Based on the newly discovered role of $\mathrm{Cdk} 5 \mathrm{in} \mathrm{Rb}$ regulation, the question is arising as whether Cdk5 acts as an alternate driver in Cdk4/6-resistant cancers, broadening the spectrum for treatment strategies while increasing the urgency for moving Cdk5 specific anticancer drugs into the clinical arsenal. In this upcoming era of personalized medicine, defining a set of biomarkers that differentiate Cdk5from Cdk4/6-driven cancers will be crucial to match cancer patients with the appropriate treatment. 


\section{Acknowledgments}

This work was supported by a North American Neuroendocrine Tumor Society fellowship (KP), U.S. National Institutes of Health Grants to J.A.B. (MH79710, MH083711, DA016672, DA033485, and NS073855) and American Cancer Society MEN2 Thyroid Cancer Consortium Research Grant (RSGM-11-190-01, J.A.B.). We thank Erik Knudsen and Florian Plattner for helpful discussions and Kate Mackley for graphic art. We apologize to authors whose work was not cited due to space limitations. Some images are used under license from Shutterstock.com. Credits to S. Kaulitzki/S K Chavan/M. Vaculikova/J. Vitanovski/Shutterstock.

\section{References}

1. Hanahan D, Weinberg RA. Hallmarks of cancer: the next generation. Cell. 2011; 144:646-674. [PubMed: 21376230]

2. Asghar U, et al. The history and future of targeting cyclin-dependent kinases in cancer therapy. Nat Rev Drug Discov. 2015; 14:130-146. [PubMed: 25633797]

3. Dhavan R, Tsai LH. A decade of CDK5. Nat Rev Mol Cell Biol. 2001; 2:749-759. [PubMed: 11584302]

4. Lockwood WW, et al. DNA amplification is a ubiquitous mechanism of oncogene activation in lung and other cancers. Oncogene. 2008; 27:4615-4624. [PubMed: 18391978]

5. Harada T, et al. Genome-wide DNA copy number analysis in pancreatic cancer using high-density single nucleotide polymorphism arrays. Oncogene. 2008; 27:1951-1960. [PubMed: 17952125]

6. Eggers JP, et al. Cyclin-dependent kinase 5 is amplified and overexpressed in pancreatic cancer and activated by mutant K-Ras. Clin Cancer Res. 2011; 17:6140-6150. [PubMed: 21825040]

7. Choi HS, et al. Single-nucleotide polymorphisms in the promoter of the CDK5 gene and lung cancer risk in a Korean population. J Hum Genet. 2009; 54:298-303. [PubMed: 19343042]

8. Kibel AS, et al. Genetic variants in cell cycle control pathway confer susceptibility to aggressive prostate carcinoma. The Prostate. 2016; 76:479-490. [PubMed: 26708993]

9. Wei K, et al. An immunohistochemical study of cyclin-dependent kinase 5 (CDK5) expression in non-small cell lung cancer (NSCLC) and small cell lung cancer (SCLC): a possible prognostic biomarker. World J Surg Oncol. 2016; 14:34. [PubMed: 26860827]

10. Meder L, et al. NOTCH, ASCL1, p53 and RB alterations define an alternative pathway driving neuroendocrine and small cell lung carcinomas. Int J Cancer. 2016; 138:927-938. [PubMed: 26340530]

11. Liu JL, et al. Cyclin-dependent kinase 5 regulates the proliferation, motility and invasiveness of lung cancer cells through its effects on cytoskeletal remodeling. Mol Med Rep. 2015; 12:39793985. [PubMed: 26018459]

12. Pozo K, et al. The role of Cdk5 in neuroendocrine thyroid cancer. Cancer Cell. 2013; 24:499-511. [PubMed: 24135281]

13. Xie W, et al. Phosphorylation of kinase insert domain receptor by cyclin-dependent kinase 5 at serine 229 is associated with invasive behavior and poor prognosis in prolactin pituitary adenomas. Oncotarget. 2016

14. Liu JL, et al. Expression of CDK5/p35 in resected patients with non-small cell lung cancer: relation to prognosis. Med Oncol. 2011; 28:673-678. [PubMed: 20354813]

15. Liang Q, et al. CDK5 is essential for TGF-beta1-induced epithelial-mesenchymal transition and breast cancer progression. Sci Rep. 2013; 3:2932. [PubMed: 24121667]

16. Chiker $\mathrm{S}$, et al. Cdk5 promotes DNA replication stress checkpoint activation through RPA-32 phosphorylation, and impacts on metastasis free survival in breast cancer patients. Cell Cycle. 2015; 14:3066-3078. [PubMed: 26237679]

17. Catania A, et al. Expression and localization of cyclin-dependent kinase 5 in apoptotic human glioma cells. Neuro Oncol. 2001; 3:89-98. [PubMed: 11296485]

18. Yushan R, et al. Insights into the clinical value of cyclin-dependent kinase 5 in glioma: a retrospective study. World J Surg Oncol. 2015; 13:223. [PubMed: 26205145]

19. Zhang X, et al. Aberrant expression of CDK5 infers poor outcomes for nasopharyngeal carcinoma patients. Int J Clin Exp Pathol. 2015; 8:8066-8074. [PubMed: 26339373] 
20. Cao L, et al. Cyclin-dependent kinase 5 decreases in gastric cancer and its nuclear accumulation suppresses gastric tumorigenesis. Clin Cancer Res. 2015; 21:1419-1428. [PubMed: 25609066]

21. Lu JW, et al. Decreased expression of $\mathrm{p} 39$ is associated with a poor prognosis in human hepatocellular carcinoma. Med Oncol. 2011; 28(Suppl 1):S239-245. [PubMed: 20936377]

22. Lopes JP, Agostinho P. Cdk5: multitasking between physiological and pathological conditions. Prog Neurobiol. 2011; 94:49-63. [PubMed: 21473899]

23. Futatsugi A, et al. Cyclin-dependent kinase 5 regulates E2F transcription factor through phosphorylation of Rb protein in neurons. Cell Cycle. 2012; 11:1603-1610. [PubMed: 22456337]

24. Ogara MF, et al. CDK5-mediated phosphorylation of p19INK4d avoids DNA damage-induced neurodegeneration in mouse hippocampus and prevents loss of cognitive functions. Biochim Biophys Acta. 2014; 1843:1309-1324. [PubMed: 24703879]

25. Chang KH, et al. Deregulated Cdk5 triggers aberrant activation of cell cycle kinases and phosphatases inducing neuronal death. J Cell Sci. 2012; 125:5124-5137. [PubMed: 22899714]

26. Fu AK, et al. Cyclin-dependent kinase 5 phosphorylates signal transducer and activator of transcription 3 and regulates its transcriptional activity. Proc Natl Acad Sci U S A. 2004; 101:6728-6733. [PubMed: 15096606]

27. Lin $\mathrm{H}$, et al. Cdk5 regulates STAT3 activation and cell proliferation in medullary thyroid carcinoma cells. J Biol Chem. 2007; 282:2776-2784. [PubMed: 17145757]

28. Hsu FN, et al. Cyclin-dependent kinase 5 modulates STAT3 and androgen receptor activation through phosphorylation of Ser(7)(2)(7) on STAT3 in prostate cancer cells. Am J Physiol Endocrinol Metab. 2013; 305:E975-986. [PubMed: 23941877]

29. Lin $\mathrm{H}$, et al. Involvement of Cdk5/p25 in digoxin-triggered prostate cancer cell apoptosis. J Biol Chem. 2004; 279:29302-29307. [PubMed: 15123618]

30. Lindqvist J, et al. Cyclin-dependent kinase 5 acts as a critical determinant of AKT-dependent proliferation and regulates differential gene expression by the androgen receptor in prostate cancer cells. Mol Biol Cell. 2015; 26:1971-1984. [PubMed: 25851605]

31. Goldstein M, Kastan MB. The DNA damage response: implications for tumor responses to radiation and chemotherapy. Annu Rev Med. 2015; 66:129-143. [PubMed: 25423595]

32. Turner NC, et al. A synthetic lethal siRNA screen identifying genes mediating sensitivity to a PARP inhibitor. EMBO J. 2008; 27:1368-1377. [PubMed: 18388863]

33. Courapied S, et al. The cdk5 kinase regulates the STAT3 transcription factor to prevent DNA damage upon topoisomerase I inhibition. J Biol Chem. 2010; 285:26765-26778. [PubMed: 20516069]

34. Ehrlich SM, et al. Targeting cyclin dependent kinase 5 in hepatocellular carcinoma--A novel therapeutic approach. J Hepatol. 2015; 63:102-113. [PubMed: 25660209]

35. Yu HP, et al. TIGAR regulates DNA damage and repair through pentosephosphate pathway and Cdk5-ATM pathway. Sci Rep. 2015; 5:9853. [PubMed: 25928429]

36. Lee JH, Kim KT. Regulation of cyclin-dependent kinase 5 and p53 by ERK1/2 pathway in the DNA damage-induced neuronal death. J Cell Physiol. 2007; 210:784-797. [PubMed: 17117479]

37. Tian B, et al. Phosphorylation of ATM by Cdk5 mediates DNA damage signalling and regulates neuronal death. Nat Cell Biol. 2009; 11:211-218. [PubMed: 19151707]

38. Vigneron A, et al. The EGFR-STAT3 oncogenic pathway up-regulates the Eme 1 endonuclease to reduce DNA damage after topoisomerase I inhibition. Cancer Res. 2008; 68:815-825. [PubMed: 18245483]

39. Bolin C, et al. The impact of cyclin-dependent kinase 5 depletion on poly(ADP-ribose) polymerase activity and responses to radiation. Cell Mol Life Sci. 2012; 69:951-962. [PubMed: 21922195]

40. Zhang F, et al. Enhancement of radiosensitivity by roscovitine pretreatment in human non-small cell lung cancer A549 cells. J Radiat Res. 2008; 49:541-548. [PubMed: 18728343]

41. Bhandari D, et al. Cyclin-dependent kinase 5 activates guanine nucleotide exchange factor GIV/ Girdin to orchestrate migration-proliferation dichotomy. Proc Natl Acad Sci U S A. 2015; 112:E4874-4883. [PubMed: 26286990]

42. Demelash A, et al. Achaete-scute homologue-1 (ASH1) stimulates migration of lung cancer cells through Cdk5/p35 pathway. Mol Biol Cell. 2012; 23:2856-2866. [PubMed: 22696682] 
43. Strock CJ, et al. Cyclin-dependent kinase 5 activity controls cell motility and metastatic potential of prostate cancer cells. Cancer Res. 2006; 66:7509-7515. [PubMed: 16885348]

44. Feldmann G, et al. Cyclin-dependent kinase inhibitor Dinaciclib (SCH727965) inhibits pancreatic cancer growth and progression in murine xenograft models. Cancer Biol Ther. 2011; 12:598-609. [PubMed: 21768779]

45. Bisht S, et al. Cyclin-Dependent Kinase 5 (CDK5) Controls Melanoma Cell Motility, Invasiveness, and Metastatic Spread-Identification of a Promising Novel therapeutic target. Transl Oncol. 2015; 8:295-307. [PubMed: 26310376]

46. Herzog J, et al. Cyclin-dependent kinase 5 stabilizes hypoxia-inducible factor-1alpha: a novel approach for inhibiting angiogenesis in hepatocellular carcinoma. Oncotarget. 2016; 7:2710827121. [PubMed: 27027353]

47. Sharma MR, et al. Angiostatin-induced inhibition of endothelial cell proliferation/apoptosis is associated with the down-regulation of cell cycle regulatory protein cdk5. J Cell Biochem. 2004; 91:398-409. [PubMed: 14743398]

48. Liebl J, et al. Cyclin-dependent kinase 5 regulates endothelial cell migration and angiogenesis. J Biol Chem. 2010; 285:35932-35943. [PubMed: 20826806]

49. Merk $\mathrm{H}$, et al. Inhibition of endothelial Cdk5 reduces tumor growth by promoting non-productive angiogenesis. Oncotarget. 2016; 7:6088-6104. [PubMed: 26755662]

50. de Nigris F, et al. Osteosarcoma cells induce endothelial cell proliferation during neo-angiogenesis. Journal of Cellular Physiology. 2013; 228:846-852. [PubMed: 23042366]

51. Weitensteiner SB, et al. Trisubstituted pyrazolopyrimidines as novel angiogenesis inhibitors. PLoS One. 2013; 8:e54607. [PubMed: 23336010]

52. Liebl J, et al. Cdk5 controls lymphatic vessel development and function by phosphorylation of Foxc2. Nat Commun. 2015; 6:7274. [PubMed: 26027726]

53. Peyressatre M, et al. Targeting cyclin-dependent kinases in human cancers: from small molecules to Peptide inhibitors. Cancers (Basel). 2015; 7:179-237. [PubMed: 25625291]

54. Cicenas J, et al. Roscovitine in cancer and other diseases. Ann Transl Med. 2015; 3:135. [PubMed: 26207228]

55. Karran E, Palmer AM. Neurodegenerative disorders and their treatment. Drug News Perspect. 2007; 20:407-412. [PubMed: 17925895]

56 . Weishaupt $\mathrm{JH}$, et al. Inhibition of CDK5 is protective in necrotic and apoptotic paradigms of neuronal cell death and prevents mitochondrial dysfunction. Mol Cell Neurosci. 2003; 24:489 502. [PubMed: 14572469]

57. Plattner F, et al. Memory enhancement by targeting Cdk5 regulation of NR2B. Neuron. 2014; 81:1070-1083. [PubMed: 24607229]

58. Plattner F, et al. The role of ventral striatal cAMP signaling in stress-induced behaviors. Nat Neurosci. 2015; 18:1094-1100. [PubMed: 26192746]

59. Wissing MD, et al. Small-molecule screening of PC3 prostate cancer cells identifies tilorone dihydrochloride to selectively inhibit cell growth based on cyclin-dependent kinase 5 expression. Oncol Rep. 2014; 32:419-424. [PubMed: 24841903]

60. Shi Z, et al. AC1MMYR2, an inhibitor of dicer-mediated biogenesis of Oncomir miR-21, reverses epithelial-mesenchymal transition and suppresses tumor growth and progression. Cancer Res. 2013; 73:5519-5531. [PubMed: 23811941]

61. Ren Y, et al. AC1MMYR2 impairs high dose paclitaxel-induced tumor metastasis by targeting miR-21/CDK5 axis. Cancer Lett. 2015; 362:174-182. [PubMed: 25827073]

62. Dorand RD, et al. Cdk5 disruption attenuates tumor PD-L1 expression and promotes antitumor immunity. Science. 2016; 353:399-403. [PubMed: 27463676]

63. Liebl J, et al. Twice switched at birth: cell cycle-independent roles of the "neuron-specific" cyclindependent kinase 5 (Cdk5) in non-neuronal cells. Cell Signal. 2011; 23:1698-1707. [PubMed: 21741478]

64. Arif A. Extraneuronal activities and regulatory mechanisms of the atypical cyclin-dependent kinase Cdk5. Biochem Pharmacol. 2012; 84:985-993. [PubMed: 22795893] 
65. Brinkkoetter PT, et al. Cyclin I activates Cdk5 and regulates expression of Bcl-2 and Bcl-XL in postmitotic mouse cells. The Journal of Clinical Investigation. 2009; 119:3089-3101. [PubMed: 19729834]

66. Li R, et al. Cyclin I promotes cisplatin resistance via Cdk5 activation in cervical cancer. Eur Rev Med Pharmacol Sci. 2015; 19:4533-4541. [PubMed: 26698249]

67. Hisanaga $\mathrm{S}$, Saito T. The regulation of cyclin-dependent kinase 5 activity through the metabolism of p35 or p39 Cdk5 activator. Neurosignals. 2003; 12:221-229. [PubMed: 14673209]

68. Lindqvist J, et al. Studying Nestin and its Interrelationship with Cdk5. Methods Enzymol. 2016; 568:509-535. [PubMed: 26795482]

69. Takashima A, et al. Involvement of cyclin dependent kinase 5 activator p 25 on tau phosphorylation in mouse brain. Neurosci Lett. 2001; 306:37-40. [PubMed: 11403952]

70. Rouzier R, et al. Microtubule-associated protein tau: A marker of paclitaxel sensitivity in breast cancer. Proceedings of the National Academy of Sciences of the United States of America. 2005; 102:8315-8320. [PubMed: 15914550]

71. Grant NJ, et al. Phosphorylation of a splice variant of collapsin response mediator protein 2 in the nucleus of tumour cells links cyclin dependent kinase-5 to oncogenesis. BMC Cancer. 2015; 15:885. [PubMed: 26555036]

72. Hayashi K, et al. Phosphorylation of the tubulin-binding protein, stathmin, by Cdk5 and MAP kinases in the brain. Journal of Neurochemistry. 2006; 99:237-250. [PubMed: 16925597]

73. Nie W, et al. Overexpression of stathmin 1 is a poor prognostic biomarker in non-small cell lung cancer. Lab Invest. 2015; 95:56-64. [PubMed: 25384122]

74. Huang C, et al. Talin phosphorylation by Cdk5 regulates Smurf1-mediated talin head ubiquitylation and cell migration. Nat Cell Biol. 2009; 11:624-630. [PubMed: 19363486]

75. Quintavalle M, et al. A cell-based high-content screening assay reveals activators and inhibitors of cancer cell invasion. Sci Signal. 2011; 4:ra49. [PubMed: 21791703]

76. Zhang S, et al. CDK5 Regulates Paclitaxel Sensitivity in Ovarian Cancer Cells by Modulating AKT Activation, p21Cip1- and p27Kip1-Mediated G1 Cell Cycle Arrest and Apoptosis. PLoS One. 2015; 10:e0131833. [PubMed: 26146988]

77. Mimori K, et al. Reduced tau expression in gastric cancer can identify candidates for successful Paclitaxel treatment. Br J Cancer. 2006; 94:1894-1897. [PubMed: 16721363]

78. Kim E, et al. CDK5 is a novel regulatory protein in PPARgamma ligand-induced antiproliferation. Int J Oncol. 2006; 28:191-194. [PubMed: 16327995]

79. Lowman XH, et al. The proapoptotic function of Noxa in human leukemia cells is regulated by the kinase Cdk5 and by glucose. Mol Cell. 2010; 40:823-833. [PubMed: 21145489]

80. Leshchenko VV, et al. Genomewide DNA methylation analysis reveals novel targets for drug development in mantle cell lymphoma. Blood. 2010; 116:1025-1034. [PubMed: 20427703]

81. Levacque Z, et al. Level of cdk5 expression predicts the survival of relapsed multiple myeloma patients. Cell Cycle. 2012; 11:4093-4095. [PubMed: 22987154]

82. Hsu FN, et al. Regulation of androgen receptor and prostate cancer growth by cyclin-dependent kinase 5. J Biol Chem. 2011; 286:33141-33149. [PubMed: 21799006]

83. Huang E, et al. The role of Cdk5-mediated apurinic/apyrimidinic endonuclease 1 phosphorylation in neuronal death. Nat Cell Biol. 2010; 12:563-571. [PubMed: 20473298]

84. Tripathi BK, et al. CDK5 is a major regulator of the tumor suppressor DLC1. J Cell Biol. 2014; 207:627-642. [PubMed: 25452387]

85. Xie Z, et al. Serine 732 phosphorylation of FAK by Cdk5 is important for microtubule organization, nuclear movement, and neuronal migration. Cell. 2003; 114:469-482. [PubMed: 12941275]

86. Filippova N, et al. Phosphoregulation of the RNA-binding Protein Hu Antigen R (HuR) by Cdk5 Affects Centrosome Function. Journal of Biological Chemistry. 2012; 287:32277-32287. [PubMed: 22829587]

87. Rashid T, et al. Phosphorylation of Pak1 by the p35/Cdk5 kinase affects neuronal morphology. J Biol Chem. 2001; 276:49043-49052. [PubMed: 11604394] 
88. Lee JH, et al. Stabilization and activation of $\mathrm{p} 53$ induced by Cdk5 contributes to neuronal cell death. J Cell Sci. 2007; 120:2259-2271. [PubMed: 17591690]

89. Ajay AK, et al. Cdk5 phosphorylates non-genotoxically overexpressed p53 following inhibition of PP2A to induce cell cycle arrest/apoptosis and inhibits tumor progression. Mol Cancer. 2010; 9:204. [PubMed: 20673369]

90. Liu R, et al. Cdk5-mediated regulation of the PIKE-A-Akt pathway and glioblastoma cell invasion. Proc Natl Acad Sci U S A. 2008; 105:7570-7575. [PubMed: 18487454]

91. Lee KY, et al. Neuronal Cdc2-like kinase (Nclk) binds and phosphorylates the retinoblastoma protein. J Biol Chem. 1997; 272:5622-5626. [PubMed: 9038171]

92. Xie W, et al. CDK5 and its activator P35 in normal pituitary and in pituitary adenomas: relationship to VEGF expression. Int J Biol Sci. 2014; 10:192-199. [PubMed: 24550687] 


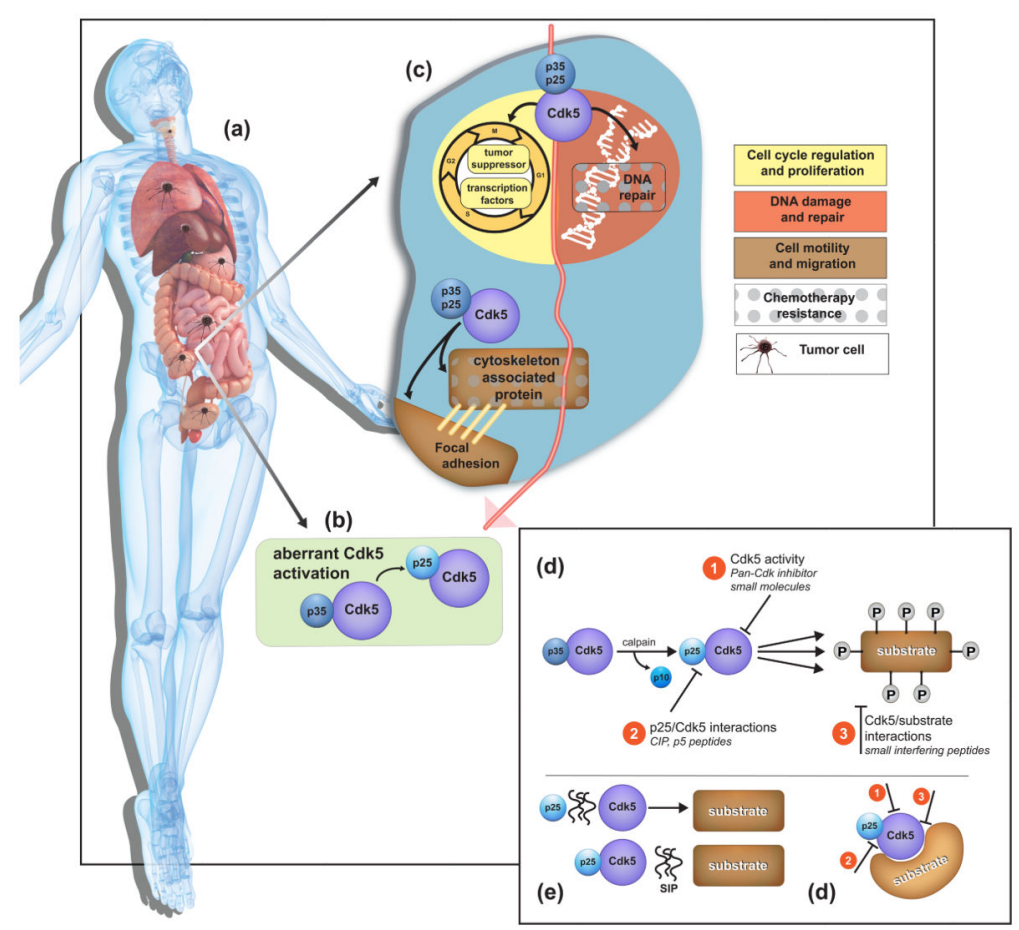

Figure 1 - Key Figure. Targeting Cdk5 in cancer

(a) Cdk5 contributes to carcinogenesis in several organs throughout the body. (b) Cdk5 activation is dependent on its binding to the cofactor, $\mathrm{p} 35$, or its proteolytic cleavage product, p25 (green box). (c) At a cellular level, Cdk5 is involved in the regulation of the cell cycle and cell proliferation by phosphorylating tumor suppressors and transcription factors, and in the DNA damage response upon exposure to genotoxic agents such as chemotherapy and radiotherapy. $\mathrm{Cdk} 5$ plays a role in cell motility and migration by regulating the cytoskeleton and focal adhesions. The role of $\mathrm{Cdk} 5$ in the DNA damage response and cytoskeleton remodeling has been linked to resistance to common chemotherapies.

Therapeutic targeting of Cdk5 is achieved either by 1) inhibiting Cdk5 kinase activity with a pan-Cdk inhibitor or small molecules (d); by 2) preventing Cdk5 binding to p25 using peptides (e); or by 3 ) interfering with $\mathrm{Cdk} 5$ association and phosphorylation of its substrate using peptides (f). 


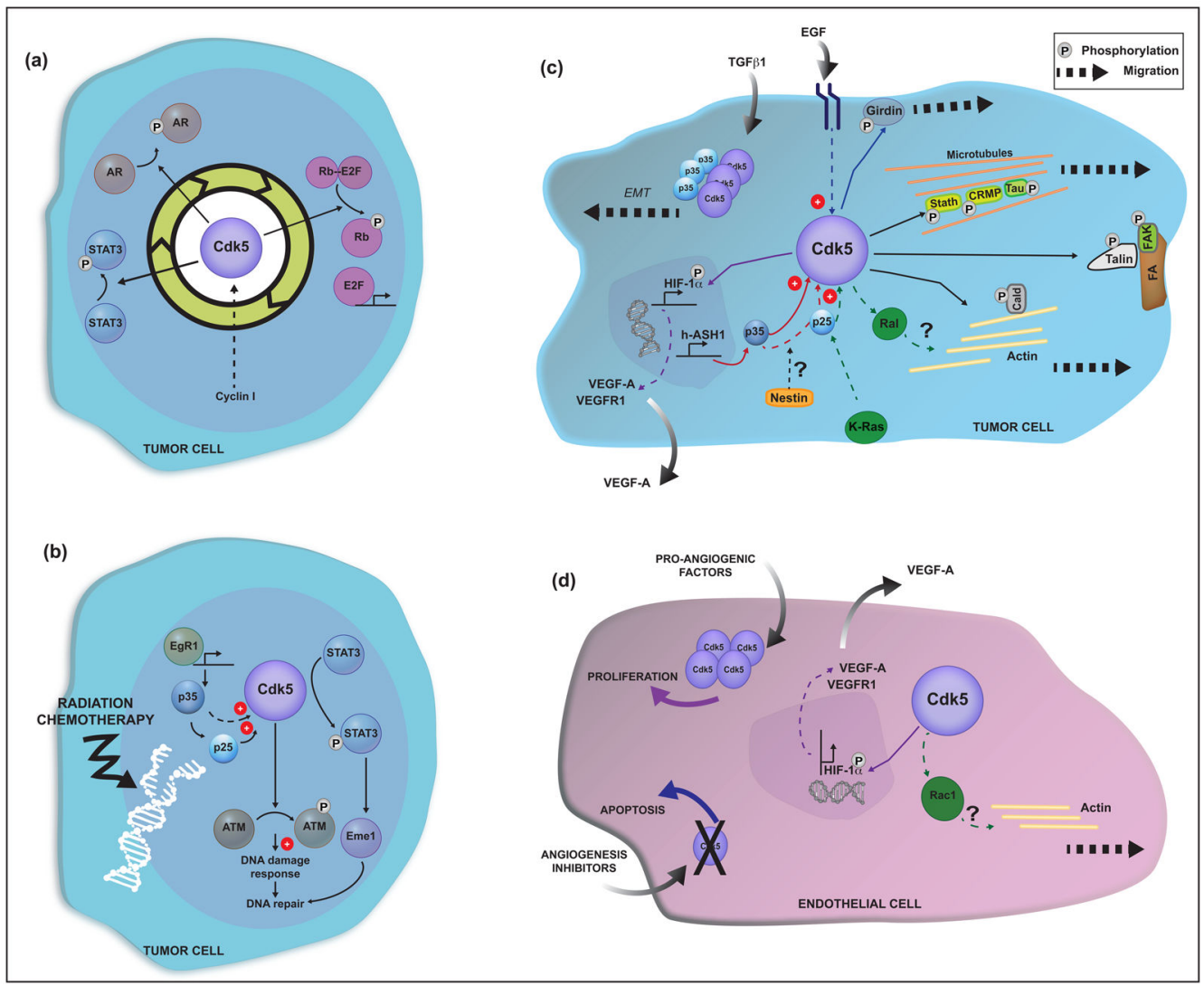

Figure 2. Cdk5-driven mechanisms in cancer progression

(a) Schematic representation of Cdk5 contribution to cell cycle and proliferation. Cdk5 phosphorylates tumor suppressors and transcription factors involved in cell cycle progression. Cyclin I may bind and activate Cdk5 during the cell cycle. The phases at which Cdk5 regulates each transcription factor have not been clearly defined. (b) Proposed role for Cdk5 in the DNA damage response and DNA repair processes. Cdk5 becomes activated in tumor cells exposed to DNA damaging agents (i.e. radiation and chemotherapy). EgR1 induces p35 expression, which binds and activates Cdk5. P35 can be cleaved to produce p25, which activates $\mathrm{Cdk} 5$, as in brain cancers. Upon activation, Cdk5 phosphorylates the checkpoint kinase ATM and the transcription factor STAT3 to transduce DNA damage responses and facilitate DNA repair. (c) Cdk5 in cell motility and migration. Cdk5 becomes activated upon stimulation with TGF $\beta$ or EGF and phosphorylates components of the cytoskeleton and focal adhesions to induce cell motility and migration. Cdk5 may facilitate neoplasia and angiogenesis through HIF1 $\alpha$ and VEGF signaling. Induction of hAsh1 and subsequent expression of p35 may be a mechanism by which Cdk5 is activated as it occurs in lung cancer. The intermediate filament protein, nestin, is a Cdk5 substrate and might regulate the cleavage of p35-to-p25 by an autoregulatory process. (d) Role of Cdk5 in endothelial cell proliferation and migration. Cdk5 is expressed in endothelial cells, is activated by pro-angiogenic factors and regulates angiogenesis by facilitating the migration of endothelial cells via a Rac-dependent mechanism.

Abbreviations- Retinoblastoma protein (Rb), E2 transcription factor (E2F), androgen receptor (AR), signal transducer and activator of transcription 3 (STAT3), Cdk5-mediated 
phosphorylation $(\mathrm{P})$, early growth response protein (EgR1), Ataxia-telangiectasia mutated (ATM), DNA endonuclease (Eme1), human achaete-scute homolog 1 (hASH1), Epithelialto-mesenchymal transition (EMT), hypoxia-induced factor 1a (HIF1a), Stathmin (Stath), Collapsin Response Mediator Protein-2 (CRMP), focal adhesions (FA), focal adhesion kinase (FAK), Caldesmon (Cald), vascular endothelial growth factor A (VEGF-A), vascular endothelial growth factor receptor 1 (VEGFR1). 


\section{Table 1}

\section{Expression of $\mathrm{Cdk} 5$ in human cancers}

\begin{tabular}{|c|c|c|}
\hline Cancer & Cdk5 function & References \\
\hline Breast & Proliferation, survival, EMT, metastasis & {$[15,16]$} \\
\hline Colorectal & Proliferation & {$[78]$} \\
\hline Gastric & Cell cycle suppressor & {$[20]$} \\
\hline Glioma, glioblastoma & Invasion & {$[17,18]$} \\
\hline Hepatocellular carcinoma & Migration, angiogenesis, inflammation, lymphatic development & {$[21,34]$} \\
\hline Head and Neck - & Not known & {$[19]$} \\
\hline Leukemia & Survival & [79] \\
\hline Lung - Small cell lung cancer (SCLC), carcinoids & Proliferation, migration & {$[42]$} \\
\hline Lung - Non small cell lung cancer (NSCLC) & Proliferation, migration, invasion & {$[4,14]$} \\
\hline Mantle cell lymphoma & Proliferation & {$[80]$} \\
\hline Medulloblastoma & Immune evasion & {$[62]$} \\
\hline Melanoma & Proliferation, invasion & {$[45]$} \\
\hline Multiple myeloma & Not known & {$[81]$} \\
\hline Ovary & Migration & {$[19]$} \\
\hline Pancreatic & Proliferation, migration, invasion, metastasis & {$[6,44]$} \\
\hline Pituitary Adenoma & Migration, invasion & {$[13]$} \\
\hline Prostate & Proliferation, migration, invasion, metastasis, apoptosis & {$[43]$} \\
\hline Thyroid - Medullary thyroid carcinoma & Proliferation & {$[12]$} \\
\hline
\end{tabular}




\section{Table 2}

Cdk5 substrates implicated in cancer

\begin{tabular}{llll}
\hline Substrate & Site & Protein type/Cellular function & References \\
\hline Akt & Ser-473 & Kinase/proliferation & {$[30]$} \\
\hline AR & Ser-81, Ser-308 & Transcription factor/proliferation & {$[82]$} \\
\hline Ape1 & Thr-232 & Endonuclease/DNA Damage Response & {$[83]$} \\
\hline ATM & & Kinase/DNA Damage Response & {$[37]$} \\
\hline Caldesmon & Ser-527 & Cytoskeletal protein/Migration & {$[75]$} \\
\hline DLC-1 & Ser-120, Ser-205, Ser-422, Ser-509 & Tumor suppressor/Proliferation & {$[84]$} \\
\hline FAK & Ser-732 & Cytoskeletal protein/Migration/EMT & {$[15,85]$} \\
\hline Girdin & Ser-1674 & GTPase/Migration & {$[41]$} \\
\hline Hu antigen R & Ser-202 & mRNA binding protein/Proliferation & {$[86]$} \\
\hline Noxa & Ser-15 & Pro-apoptotic factor/Apoptosis & {$[79]$} \\
\hline PAK1 & Thr-212 & Cytoskeletal protein/Migration & {$[87]$} \\
\hline p53 & Ser-15, Ser-33, Ser-46 & Tumor suppressor/Apoptosis & {$[88]$} \\
\hline PIKE-A & Ser-279 & GTPase/Migration, invasion & {$[90]$} \\
\hline Rb & Ser-780, Ser-795, Ser-807/811 & Tumor suppressor/Proliferation & {$[91]$} \\
\hline STAT-3 & Ser-727 & Transcription factor/Proliferation & {$[26]$} \\
\hline Talin & Ser-425 & Cytoskeletal protein/Migration & {$[74]$} \\
\hline VEGFR-2 & Ser-229 & Tyrosine kinase recptor/Invasion & {$[92]$} \\
\hline
\end{tabular}

